\title{
Atuação fisioterapêutica em gestantes com COVID-19 em ambiente hospitalar: Uma revisão integrativa
}

\author{
Physiotherapeutic practice in pregnant women with COVID-19 in a hospital environment: An \\ integrative review
}

Desempeño fisioterapéutico en mujeres embarazadas con COVID-19 en un entorno hospitalario: Una revisión integrativa

Recebido: 24/05/2021 | Revisado: 01/06/2021 | Aceito: 02/06/2021 | Publicado: 18/06/2021

\author{
Amanda Fernandes Pacheco \\ ORCID: https://orcid.org/0000-0001-9796-1972 \\ Universidade Federal do Pampa, Brasil \\ E-mail: amandafp2005@ hotmail.com \\ Fabiana Vargas-Ferreira \\ ORCID: https://orcid.org/0000-0003-3567-4737 \\ Universidade Federal de Minas Gerais, Brasil \\ E-mail: fabivfer@yahoo.com.br \\ Fernanda Vargas Ferreira \\ ORCID: https://orcid.org/0000-0002-2009-3422 \\ Universidade Federal do Pampa, Brasil \\ E-mail: fernandaferreira@unipampa.edu.br
}

\begin{abstract}
Resumo
Introdução: Durante o ciclo gravídico-puerperal ocorrem alterações fisiológicas que parecem promover quadro clínico grave devido à COVID-19. O objetivo do estudo foi identificar evidências científicas sobre as abordagens fisioterapêuticas realizadas em gestantes com COVID-19 em ambiente hospitalar. Método: Revisão integrativa da literatura realizada por meio do acesso às bases de dados BVS, Embase e MEDLINE com descritores, respectivamente, do DECS, Emtree e MESH em abril de 2021. Foram incluídos artigos oriundos de pesquisa original, na íntegra, em inglês, português ou espanhol, no recorte temporal de 2020 a março de 2021 e que apresentassem o desfecho primário. Resultados: Encontraram-se três artigos que empregaram condutas fisioterapêuticas direcionadas a gestantes com COVID-19. As principais complicações decorrentes da COVID-19 foram respiratórias e neurológicas. Foram empregadas técnicas respiratórias, mobilização precoce progressiva, exercícios terapêuticos, estímulo a posições verticalizadas e à deambulação. Conclusão: Assim, a revisão integrativa mostrou a importância do atendimento fisioterapêutico em ambiente hospitalar voltado a gestantes. Sugere-se que haja maior divulgação da atuação fisioterapêutica, especialmente, no que tange ao risco elevado de complicações no ciclo gravídico-puerperal e ao objetivo primário - recuperação funcional.
\end{abstract}

Palavras-chave: COVID-19; Gestação; Modalidades de fisioterapia; Revisão.

\begin{abstract}
Introduction: During the pregnancy-puerperium cycle there are physiological changes which seem to promote a severe clinical presentation due to COVID-19. The purpose of this study was to identify scientifical productions in the literature regarding physiotherapeutic practice in pregnant women with COVID-19 in a hospital environment. Method: It is an integrative review conducted through access to VHL, Embase and MEDLINE databases using the keywords, respectively, from DECS, Emtree e MESH in 2021 april. Full articles in English, Portuguese or Spanish from original researches were included in the time cut from 2020 to 2021 march, and which presented the outcome. Results: Three articles highlighting the issue were found which applied therapeutic strategies targeted at pregnant women with COVID-19. Respiratory symptoms and neurological manifestations were the main complications due to COVID-19. Breathing techniques, early progressive mobilization, therapeutic exercises, vertical positions and ambulation were applied. Conclusion: Thus, this integrative review showed the importance of physiotherapeutic practice in pregnant women with COVID-19 in a hospital environment. Further dissemination of the field of Physiotherapy, especially, related to high-risk of complications in pregnancy and postpartum and to the main outcome - functional recovery.
\end{abstract}

Keywords: COVID-19; Pregnancy; Physical therapy Specialty; Review.

\section{Resumen}

Introducción: Durante el ciclo embarazo-puerperal hay cambios fisiológicos que parecen promover un cuadro clínico severo debido al COVID-19. El objetivo era identificar evidencia científica sobre enfoques de fisioterapia realizados 
en mujeres embarazadas con COVID-19 en un entorno hospitalario. Método: Revisión integrativa de la literatura realizada a través del acceso a las bases de datos BVS, Embase y MEDLINE con descriptores, respectivamente, de DECS, Emtree y MESH en abril de 2021. Artículos de la investigación original, íntegramente, en inglés, portugués o español, en el período de 2020 a marzo de 2021 y que presentó el resultado primario. Resultados: Se encontraron tres artículos que utilizaban abordajes fisioterapéuticos dirigidos a mujeres embarazadas con COVID-19. Las principales complicaciones derivadas del COVID-19 fueron respiratorias y neurológicas. Se utilizaron técnicas respiratorias, movilización progresiva precoz, ejercicios terapéuticos, estimulación de la posición erguida y marcha. Conclusión: Así, la revisión integradora mostró la importancia de la fisioterapia en un ambiente hospitalario para las embarazadas. Se sugiere que existe una mayor difusión de la fisioterapia, especialmente en lo que respecta al alto riesgo de complicaciones en el ciclo embarazo-puerperal y el objetivo principal - la recuperación funcional.

Palabras clave: COVID-19; Embarazo; Modalidades de fisioterapia; Revisión.

\section{Introdução}

Em janeiro de 2020, o Centro de Controle e Prevenção de Doenças da China divulgou o novo coronavírus como agente etiológico de uma doença respiratória em humanos (SARS-CoVID-2) com alto poder de contágio e rápida propagação cuja sintomatologia clínica varia de sem sintomas à presença de febre, tosse, mialgia, diarreia, vômitos e pneumonia (Ryan et al., 2020). Como fatores de risco se citam idade avançada, comorbidades, infecção HIV e doenças hepáticas/renais crônicas (Habibzadeh, Stoneman, 2020).

Na conjunção COVID-19 e gestação, as repercussões sobre a Saúde Materno-Infantil ainda não estão totalmente esclarecidas, entretanto, acredita-se que, as gestantes já são suscetíveis à infecção por agentes patógenos respiratórios (Bonham, Patterson, Strek, 2018). Tal cenário seria decorrente de uma alteração na resposta do sistema imune da gestante e na liberação de citocinas pró-inflamatórias ilustrada por aumento das concentrações plasmáticas de interleucinas 2,7 e 10 , macrófagos e fator de necrose tumoral (TNF- $\alpha$ ) (Zhang et al, 2020; Dashraath et al, 2020).

Além das repercussões pulmonares como pneumonia, fibrose e doença vascular pulmonar, as quais usualmente demandam um manejo terapêutico multimodal (George et al, 2020), complicações neurológicas e neuropsiquiátricas como acidente vascular encefálico (AVE), alteração no estado mental (eg., personalidade, comportamento, cognição) e em nível periférico (eg., raízes nervosas, junção neuromuscular) (Varatharaj et al, 2020) têm sido reportadas na literatura.

No panorama de que a doença apresenta um curso variável e que pode haver necessidade de hospitalização e/ou cuidados intensivos, especialmente, pela variedade de sintomas, como febre, dor musculoesquelética, fadiga, tosse e dificuldade respiratória (Felten-Barentsz et al, 2020), o fisioterapeuta tem sido visto como membro essencial em uma equipe multiprofissional devido à sua atuação múltipla - prevenção, reabilitação dos agravos e das disfunções geradas ao longo do processo saúde-adoecimento (Sales et al, 2020).

Ao se contextualizar com as manifestações clínicas da COVID-19, complicações obstétricas como pré-eclâmpsia, cuja prevalência ocorre entre 6 e $8 \%$ das gestações, têm aumentado devido à superposição com a infecção, incluindo elevada pressão arterial, trombocitopenia e desregulação do sistema imune (Ciobanu et al, 2016). Além disso, ambos os fatores (hipertensão e tromboctipenia) são fortes preditores de morbi-mortalidade em pessoas com COVID-19 (Moore, Suthar, 2021). Adicionalmente, ao se comparar gestantes com COVID-19 com mulheres não-grávidas também com a infecção, estudo retrospectivo mostrou que as gestantes tiveram maior taxa de hospitalização e maior tempo de permanência no hospitalar (Qeadan et al, 2021).

Considerando que gestantes são uma população de risco e que a Fisioterapia tem um papel fundamental na recuperação funcional de pessoas com COVID-19, o estudo, por meio de uma revisão integrativa, objetivou investigar quais as complicações e respectivas abordagens fisioterapêuticas realizadas em gestantes internadas em nível hospitalar. 


\section{Metodologia}

Trata-se de uma revisão integrativa de literatura baseada na formulação de pergunta norteadora "quais as complicações e abordagens fisioterapêuticas realizadas em gestantes que apresentaram a doença da COVID-19 em nível hospitalar"? Sequencialmente, realizaram-se as etapas constituintes: a) busca ou amostragem na literatura, b) coleta de dados, c) análise crítica dos estudos incluídos e d) discussão dos resultados e f) apresentação dos resultados da revisão (síntese do conhecimento) (Casarin et al, 2020).

O tema da revisão com abordagem qualitativa (Casarin et al, 2020) foi elaborado de acordo com o modelo PICOS (Population, Intervention, Comparator, Outcome, Study), conforme demonstra o Quadro 1.

Quadro 1 - Modelo PICOS empregado na revisão integrativa acerca das abordagens fisioterapêuticas realizadas em gestantes com COVID-19 em nível hospitalar, 2021.

\begin{tabular}{|c|c|}
\hline População & Gestantes \\
\hline Intervenção / Exposição & SARS-CoV-2 \\
\hline Comparação & Mulheres não-grávidas \\
\hline Desfecho & Abordagem fisioterapêutica \\
\hline Estudo & Observacional \\
\hline
\end{tabular}

Fonte: Pesquisadoras (2021).

\subsection{Estratégia de busca e seleção de estudos}

As buscas foram realizadas em abril de 2021 nas bases de dados National Library of Medicine (MEDLINE) via portal PubMed, Embase e Portal Regional da Biblioteca Virtual em Saúde (BVS).

Usaram-se as palavras-chave segundo MeSH: a) Pregnant Women, Pregnant; b) COVID-19, COVID-19 Virus Disease, COVID-19 Virus Infection, 2019-nCoV Infection, 2019-nCoV Disease, Coronavirus Disease 2019, SARS CoV 2 Infection; c) Physical Therapy Modalities, Physical Therapy Specialty e d) Hospitals. De acordo com Descritores em Ciências da Saúde (DeCs) usaram-se: a) Infecções por Coronavirus, Doença pelo Novo Coronavírus (2019-nCoV), Infecção por Coronavírus 2019-nCoV, COVID-19; b) Gravidez, Gestação; c) Fisioterapia, Modalidades de Fisioterapia e d) Assistência Hospitalar. Conforme o Emtree, empregaram-se: a) Coronavirus disease 2019; b) Pregnant woman, Pregnancy; c) Physiotherapy e d) Hospital Care. Em todas as buscas se empregaram os operadores booleano AND ou OR para a combinação desses termos.

Adotaram-se os critérios de inclusão: pesquisa original com delineamento observacional, nos idiomas espanhol, inglês ou português e publicados no recorte temporal de 2020 até março de 2021. Excluíram-se artigos que não tivessem abordagem fisioterapêutica, amostra sem gestantes e tipos de estudo (revisões, meta-análises, editoriais).

No Quadro 2 apresentam-se as bases de dados e as respectivas estratégias de busca. 
Quadro 2 - Bases de dados e estratégias de busca empregadas na revisão integrativa acerca das abordagens fisioterapêuticas realizadas em gestantes acometidas pelo novo coronavírus, 2021.

\begin{tabular}{|l|l|}
\hline Base de dados e Portais Eletrônicos & Estratégias de busca \\
\hline MEDLINE & $\begin{array}{l}\text { ("Pregnant Women" OR "Pregnant") AND ("COVID-19" OR ("COVID-19 Virus Disease" } \\
\text { OR "COVID-19 Virus Infection" OR "2019-nCoV Infection" OR "2019-nCoV Disease" OR } \\
\text { "Coronavirus Disease 2019" OR "SARS CoV 2 Infection") AND ("Physical Therapy } \\
\text { Modalities" OR "Physical Therapy Specialty") AND ("Hospitals") }\end{array}$ \\
\hline Portal Regional da BVS & $\begin{array}{l}\text { ("Infecções por Coronavirus" OR "Doença pelo Novo Coronavírus (2019-nCoV" OR } \\
\text { "Infecção por Coronavírus 2019-nCoV" OR "COVID-19") AND ("Gravidez" OR } \\
\text { "Gestação") AND ("Fisioterapia" OR "Modalidades de Fisioterapia") AND ("Assistência } \\
\text { Hospitalar") }\end{array}$ \\
\hline Embase & $\begin{array}{l}\text { ("Coronavirus disease 2019") AND ("Pregnant woman" OR "Pregnancy") AND } \\
\text { ("Physiotherapy") AND ("Hospitals") }\end{array}$ \\
\hline
\end{tabular}

Fonte: Pesquisadoras (2021).

\subsection{Extração de Dados}

Os dados foram avaliados de forma independente por duas pesquisadoras, considerando o tipo de estudo, o tamanho amostral, a idade, semanas gestacionais, complicações e encaminhamentos e/ou abordagens fisioterapêuticas. Em caso de não concordância, houve discussão para consenso.

\section{Resultados}

As buscas nas bases de dados e portais eletrônicos resultaram em 14 publicações. Em caso de duplicação, o estudo foi contabilizado somente uma vez. Excluíram-se 11 artigos (leitura de títulos e resumos). Assim, selecionaram-se 03 artigos para leitura na íntegra e avaliação da elegibilidade; tais artigos constituíram o corpus da revisão integrativa (Figura 1).

Figura 1 - Fluxograma da seleção de artigos acerca de complicações e abordagens fisioterapêuticas realizadas em gestantes infectadas pelo SARS-CoVID-2

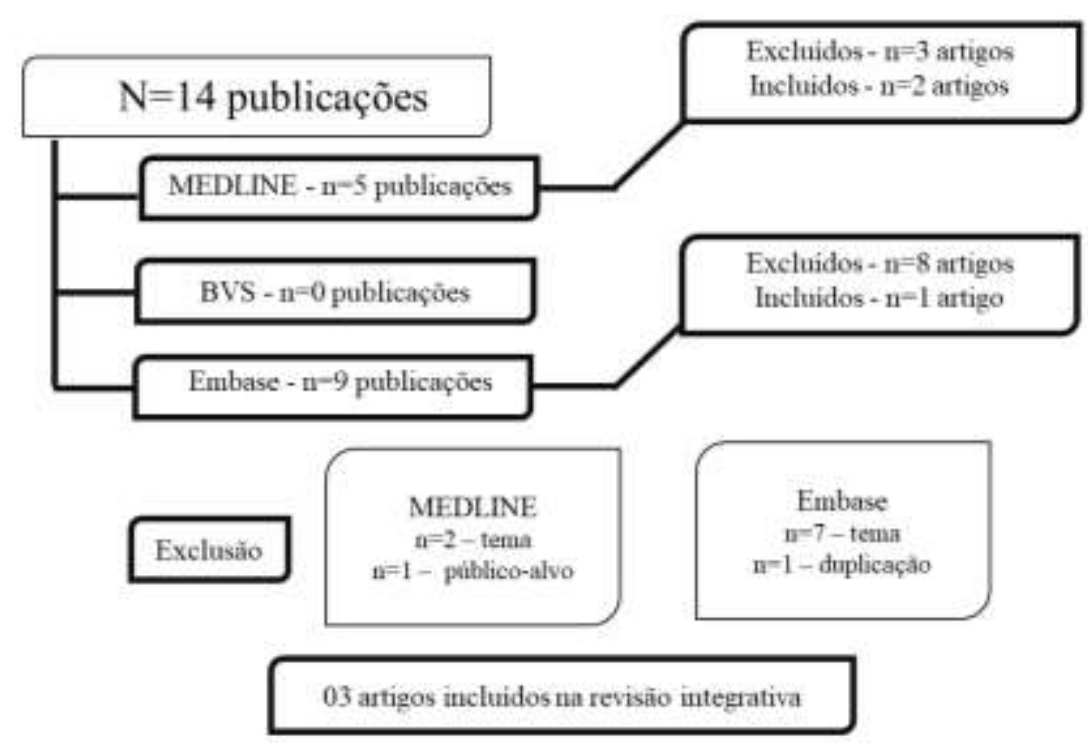

Fonte: Pesquisadoras (2021). 
No Quadro 3 constam características gerais dos artigos incluídos na revisão integrativa.

Quadro 3: Registros incluídos pela revisão integrativa, segundo título, periódico e país de origem (N=3).

\begin{tabular}{|c|c|c|c|}
\hline Citação & Títulod & Periódico $\alpha$ & País"a \\
\hline 14 & $\begin{array}{l}\text { Peripheral facial paralysis as presenting symptom of } \\
\text { COVID-19 in a pregnant woman }\end{array}$ & BMJ-Case-Rep & Brasil \\
\hline 15 & $\begin{array}{l}\text { Maintaining Mobility in a Patient Who is Pregnant and } \\
\text { Has COVID-19 Requiring Extracorporeal Membrane } \\
\text { Oxygenation: A Case Report }\end{array}$ & Phys Ther & $\begin{array}{l}\text { Estados } \\
\text { Unidos }\end{array}$ \\
\hline 16 & $\begin{array}{c}\text { Guillain Barre Syndrome following delivery in a pregnant } \\
\text { woman infected with } \text { SARS-CoV-2 }\end{array}$ & $\mathrm{J} \cdot \mathrm{Clin}$-Neurosci & Turquia \\
\hline
\end{tabular}

Fonte: Pesquisadoras (2021).

No Quadro 4 constam as descrições dos artigos incluídos na revisão integrativa (N=3). 
Quadro 4: Descrições dos artigos incluídos na revisão integrativa.

\begin{tabular}{|c|c|c|}
\hline $\mathbf{N}^{\circ}$ & Descrição do Caso & Exames, Diagnóstico Clínico e Sequência \\
\hline 14 & $\begin{array}{l}\text { Primigesta }-39^{\mathrm{a}} \text { semana } \\
\qquad 35 \text { anos } \\
\text { Sem comorbidades }\end{array}$ & $\begin{array}{l}\text { Exame neurológico: paralisia facial periférica à esquerda e sem mais particularidades (eg., } \\
\text { neuropatia ou fraqueza em membro inferior) } \\
\text { Exame laboratorial: leucocitose leve }(1,25 \times 109 \text { células/L), linfopenia }(15,2 \% ; 1,92 \times 109 \\
\text { células/L), neutrofilia (73,3\%; } 9,19 \times 109 \text { células/L), alto nível de proteína C-reativa }(61 \mathrm{mg} / \mathrm{dL}) \\
\text { Diagnóstico assumido de Paralisia de Bell } \\
\text { Testagem para SARS-CoV-2 em swab combinado de nasofaringe } \\
\text { Gestante entra em trabalho de parto - parto vaginal sem intercorrências } \\
\text { Recém-nascido testado ao nascimento e após } 48 \text { horas - SARS-CoV-2 negativo } \\
\text { Encaminhada para atendimento fisioterapêutico - leve melhora com } 15 \text { dias }\end{array}$ \\
\hline 15 & $\begin{array}{l}\text { Quadro clínico pré- } \\
\text { internação: acentuada } \\
\text { dificuldade respiratória, } \\
\text { tosse, vômito e náusea }\end{array}$ & 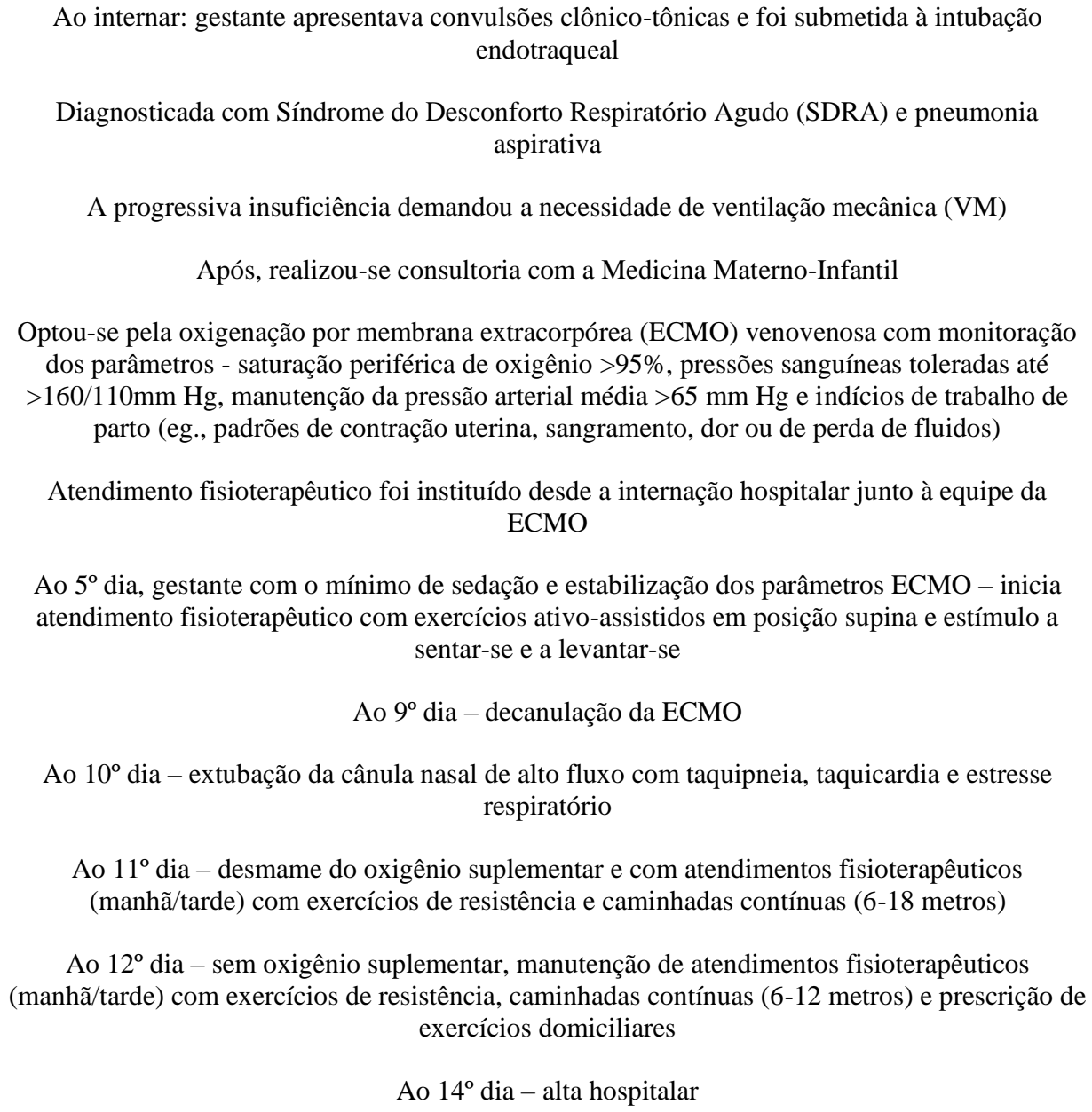 \\
\hline 16 & $\begin{array}{l}\text { Multigesta }-37^{\mathrm{a}} \text { semana } \\
\qquad 34 \text { anos } \\
\text { Sem comorbidades } \\
\text { Internada por tosse leve }\end{array}$ & $\begin{array}{c}\text { Realização de cesárea devido a sofrimento fetal } \\
\text { Alta hospitalar pós-cesárea no } 5^{\circ} \text { dia } \\
\text { Retorna no } 16^{\circ} \text { dia com dor lombar, fraqueza ascendente e sensação de formigamento em } \\
\text { membro inferior com dificuldade para deambular }\end{array}$ \\
\hline
\end{tabular}




\begin{tabular}{|c|c|}
\hline $\begin{array}{c}\text { Tomografia } \\
\text { computadorizada: } \\
\text { pneumonia consistente } \\
\text { com COVID-19 e } \\
\text { testagem para SARS- } \\
\begin{array}{c}\text { CoV-2 em swab } \\
\text { combinado de } \\
\text { nasofaringe }\end{array}\end{array}$ & $\begin{array}{r}\text { Exame neurológico: fraqueza muscular mais acentuada em membro inferior; paresia na hemiface } \\
\text { direita e sensação de formigamento nas extremidades } \\
\text { estudo da condução nervosa - diminuição da amplitude dos potenciais de ação muscular e } \\
\text { nenhuma resposta dos potenciais de ação sensorial (exceto nervo sural direito) }\end{array}$ \\
Achados consistentes com a Síndrome de Guillain-Barré (SGB) \\
Dia seguinte - apresentou dificuldade respiratória e disfagia \\
Alta hospitalar pós SGB - 12 ${ }^{\circ}$ dia \\
Encaminhamento para atendimento fisioterapêutico
\end{tabular}

Fonte: Pesquisadoras (2021)

\section{Discussão}

A revisão integrativa revelou uma escassez de publicações sobre as abordagens fisioterapêuticas em ambiente hospitalar voltadas a gestantes com infecção pelo SARS-CoVID-2. As complicações gestacionais relacionadas à COVID-19 presentes nos artigos foram distintas, o que pode sinalizar a variedade de sintomas e de possíveis sequelas pós-internação hospitalar.

Outrossim, todos os estudos foram caracterizados como relatos de caso, os quais compõem a base da pirâmide do nível de evidências nas Ciências da Saúde. Entretanto, deve-se considerar que a COVID-19 se apresenta como uma doença ainda em investigação, especialmente, no que tange à saúde materna.

Nesse sentido, o estudo de Figueiredo et al (2020) abordou a Paralisia Facial de Bell (PB) em gestante com COVID19. Essa lesão envolve o nervo facial, usualmente é monolateral e entre os possíveis fatores etiológicos se citam trauma, compressão, infecção ou inflamação (Zhang et al, 2020). Ao se contextualizar com a neuropatogênese da COVID-19, acreditase que haja o envolvimento de ACE2 (angiotensin-converting enzyme 2) que é uma proteína transmembrana expressa na superfície de diversas células do corpo como neurônios e glia. Além disso, postula-se que poderia ocorrer penetração do vírus no Sistema Nervoso Central (SNC) ou Sistema Nervoso Periférico (SNP) por via hematogênica (Ou et al, 2020).

A PB tem sido apontada como manifestação neurológica da COVID-19 cujos sinais podem aparecer no pós-parto, conforme estudo de Kumar et al (2021), no entanto, previamente à pandemia, a maioria dos casos de PB em Obstetrícia já ocorria em gestantes do último trimestre e em puérperas no período imediato, especialmente, naquelas com doenças hipertensivas específicas da gestação (eg., pré-eclâmpsia) (Ferreira et al, 2013; Shmorgun, Chan, Ray, 2002). Adicionalmente, evidências têm apontado que a abordagem fisioterapêutica apresenta efeito positivo na recuperação funcional, especialmente, frente à severidade da PB e ao tempo de recuperação (Nicastri et al, 2013), achado que foi reportado no estudo.

O relato de caso que envolveu a mobilidade materna da gestante com complicações respiratórias decorrentes da COVID-19 e com necessidade de ECMO abordou a atuação fisioterapêutica desde sua admissão até a alta hospitalar. Primeiramente, a ECMO se caracteriza como suporte mecânico temporário para o coração e os pulmões, especialmente, para pacientes com grave insuficiência respiratória e/ou cardiovascular refratárias às medidas habituais de tratamento (Peek et al, 2009). Secundariamente, a ventilação mecânica dos pacientes em ECMO deve sempre priorizar proteção pulmonar e diafragmática, bem como a estratégia ventilatória deve ser individualizada (Lages, Timenetsky, 2020).

Recente revisão sistemática que avaliou a repercussão clínica da fisioterapia em adultos submetidos à ECMO mostrou que a mobilização precoce progressiva (ortostase e deambulação) junto com técnicas respiratórias é viável para a recuperação 
funcional (Ferreira et al, 2019). Tais asserções vão ao encontro do caso clínico em que a paciente, ainda em ECMO, na posição supina realizou exercícios ativo-assistidos; no $5^{\circ}$ dia foi orientada a sentar-se e a ficar em bipedestação e, posteriormente, no $9^{\circ}$ dia a deambular no hospital.

De forma similar à PB, a Síndrome de Guillain-Barré (SGB), cuja definição é de polineuropatia inflamatória aguda com quadro clínico predominante de sintomas motores simétricos e progressivos, alterações sensitivas e acometimento da musculatura respiratória (Van den Berg et al, 2014) tem sido considerada uma manifestação neurológica da COVID-19 (Webb et al, 2020). Acredita-se que essa associação seria gerada pela reação imune excessiva advinda do SARS-CoV-2 a qual resultaria em uma "tempestade de citocinas" (eg., interleucina 6), ativando a cascata inflamatória; que por sua vez, se refletiria em danos a diferentes tecidos/sistemas, incluindo-se o respiratório e o neurológico (McGonagle et al, 2020).

Ao se contextualizar a SGB com o ciclo de vida feminino, estudo de coorte do Canadá com 16.108.819 mulheres, cujo marco inicial foi a realização de parto, foram acompanhadas entre 1989 e 2014. Entre os fatores de risco clínicos que aumentaram a chance de desenvolverem SGB se destacaram doenças reumatológicas, seguidas de autoimunes; em relação a fatores de risco obstétricos, somente a pré-eclâmpsia gerou relação positiva (Auger et al, 2018).

Dessa forma, a gestação, per se, com suas mudanças fisiológicas tais como no sistema respiratório (eg, aumento no volume corrente e redução do volume residual) e nos sistemas hematológico e cardiovascular (eg, aumento no volume plasmático e maior demanda do miocárdio) pode favorecer quadros mais graves de SGB (Pacheco et al, 2016), o que demanda uma atenção voltada à identificação precoce de sinais suspeitos como rápida e progressiva fraqueza bilateral e/ou com déficit sensorial, hiporreflexia, PB e ataxia (Leonhard et al, 2019). No caso clínico, sua primeira internação decorreu da COVID-19, período no qual se realizou uma cesárea e; posteriormente, com cerca de 15 dias, ela retornou ao hospital com sinais suspeitos de SGB, ao encontro da literatura que aponta o pós-parto como período de risco. Mais, além da abordagem fisioterapêutica para o quadro agudo, pessoas com SGB podem apresentar, a posteriori, fraqueza em membros, déficit funcional como dor crônica e fadiga (Pacheco et al, 2016), em corroboração ao relato de caso.

Embora o número de publicações tenha sido pequeno, faz-se importante comentar que a abordagem fisioterapêutica em nível hospitalar a pessoas - incluindo gestantes e puérperas - com COVID-19 compreendeu elementos de suporte respiratório/ventilatório (eg, exercícios de reexpansão torácica, treinamento muscular respiratório) e mobilização ativa (eg, mobilizações no leito, amplitude de movimento de acordo com a tolerância individual, exercícios ativo-assistidos, treinos de transferência, deambulação) (Felten-Barentsz et al, 2020). Além disso, deve-se atentar para as manifestações neurológicas que podem gerar disfunções motoras e/ou sensoriais (Gomes, Filho, Sousa, 2020),

Na perspectiva do ciclo gravídico-puerperal, urge destacar ainda que a mortalidade materna pela COVID-19 no Brasil tem sido pauta de discussão, visto que, envolve não somente aspectos biológicos / infecciosos, mas, também dificuldades na realização dos exames laboratoriais e incongruências nas políticas de saúde (Souza, Amorim, 2021). Dessa forma, o fisioterapeuta inserido em ambiente hospitalar, além de exercer sua práxis, pode juntamente com a equipe multiprofissional, auxiliar na detecção precoce de um perfil de risco de gestantes com diagnóstico positivo com base em algoritmo: a) doença leve/moderada, sem ou leve pneumonia, sinais vitais normais; sem risco para descompensação - ela pode ser liberada com orientações; caso ela apresente diabetes, hipertensão, idade $\geq 40$ anos e esteja no $3^{\circ}$ trimestre, deve-se considerar admissão; b) doença crítica/severa com hipóxia, taquipneia, dispneia, infiltração pulmonar $>50 \%$, falência respiratória e choque indicam admissão (Boushra, Koyfman, Long, 2021).

\section{Considerações Finais}

O estudo objetivou descrever as abordagens fisioterapêuticas em nível hospitalar direcionadas a gestantes com COVID-19. A amostra foi composta por três estudos que mostraram terapêuticas multimodais através de técnicas respiratórias, 
mobilização precoce progressiva (ortostase e deambulação) e exercícios terapêuticos que se mostraram viáveis e eficientes, inclusive, na presença de suporte por vida por oxigenação por membrana extracorpórea.

Isto posto, faz-se necessário a publicização da atuação fisioterapêutica hospitalar voltada à Obstetrícia, especialmente, por se tratar de uma população de risco (gestantes/puérperas), bem como no que tange à população neonatal.

\section{Agradecimentos}

Os autores agradecem aos membros do Grupo de Estudos e Pesquisa em Fisioterapia e Saúde Pélvica (GEPEFISP).

\section{Referências}

Auger, Natalie et al. Early predictors of Guillain-Barre's syndrome in the life course of women. Int J Epidemiol. 2018 Feb; 47(1): 280-288. https://doi.org/ 10.1093/ije/dyx 181 .

Bonham, C. A.; Patterson, K. C; \& Strek, M. E. Asthma outcomes and management during pregnancy. Chest. 2018 Feb;153(2):515-527. https://doi.org/10.1016/j.chest.2017.08.029.

Boushram, Marina N; Koyfman, Alex; \& Long, B. COVID-19 in pregnancy and the puerperium: A review for emergency physicians. Am J Emerg Med. 2021 Feb;40:193-198. https://doi.org/10.1016/j.ajem.2020.10.055.

Casarin, S. T. et al. Tipos de revisão de literatura: considerações das editoras do J. nurs. health. 2020;10(n.esp.):e20104031.

Ciobanu, A. M. et al. Thrombocytopenia in pregnancy. Maedica (Bucur) 2016;11:55-60. https://doi.org/10.1182/blood-2017-05-781971.

Moore, K. M; \& Suthar, M. S. Comprehensive analysis of COVID-19 during pregnancy. Biochem Biophys Res Commun. 2021 Jan 29; 538:180-186. https://doi.org/10.1016/j.bbrc.2020.12.064.

Dashraath, P. et al. Am J Obstet Gynecol. 2020 Jun;222(6):521-531. https://doi.org/10.1016/j.ajog.2020.03.021.

Felten-Barentsz, K. M. et al. Recommendations for Hospital-Based Physical Therapists Managing Patients With COVID-19. Phys Ther. 2020 Aug 31;100(9):1444-1457. https://doi.org/10.1093/ptj/pzaa114.

Ferreira, M. A. A. et al. Paralisia facial periférica e gestação: abordagem e tratamento. Rev. Bras. Ginecol. Obstet. 2013, vol.35, n.8, pp.368-372. https://doi.org/10.1590/S0100-72032013000800006.

Ferreira, D. da C. et al. Segurança e potenciais benefícios da fisioterapia em adultos submetidos ao suporte de vida com oxigenação por membrana extracorpórea: uma revisão sistemática. Rev Bras Ter Intensiva. 2019;31(2):227-239. https://doi.org/10.5935/0103-507X.20190017.

Figueiredo, R. et al. Peripheral facial paralysis as presenting symptom of COVID-19 in a pregnant woman. BMJ Case Rep 2020;13:e237146. https://doi.org/ $10.1136 /$ bcr-2020-237146.

George, P. M. et al. Respiratory follow-up of patients with COVID-19 pneumonia. Thorax 2020;0:1-8. https://doi.org/10.1136/thoraxjnl-2020-215314.

Gomes, A. de S.; Filho, O. B. de M.; Sousa, M. N. \& Alves. Associação entre o COVID-19 e manifestações neurológicas. Braz. J. of Develop., Curitiba, v. 6, n. 11, p. 88950-88961, nov. 2020. https://doi.org/10.34117/bjdv6n11-350

Habibzadeh, P.; \& Stoneman, E. K. Int J Occup Environ Med. 2020 Apr;11(2):65-71. https://doi.org/10.15171/ijoem.2020.1921.

Kumar, V. et al. Lower motor neuron facial palsy in a postnatal mother with COVID-19. BMJ Case Rep. 2021; 14(3): e240267. https://doi.org/ 10.1136/bcr2020-240267.

Lages, N. C. de L.; \& Timenetsky, K. T. Recomendações para a atuação dos fisioterapeutas nos casos de oxigenação por membrana extracorpórea (ECMO). 2020 Ago;11(Supl 1):1-276 ASSOBRAFIR Ciência. 2020 Ago;11(Supl 1):161-173.

Leonhard, S. E. et al. Diagnosis and management of Guillain-Barré syndrome in ten steps. Rev Neurol. 2019 Nov;15(11):671-683. https://doi.org/ $10.1038 / \mathrm{s} 41582-019-0250-9$.

Mark, A. et al. Maintaining Mobility in a Patient Who Is Pregnant and Has COVID-19 Requiring Extracorporeal Membrane Oxygenation: A Case Report. Phys Ther. 2021 Jan 4;101(1):pzaa189. https://doi.org/10.1093/ptj/pzaa189.

McGonagle, D. et al. The Role of Cytokines including Interleukin-6 in COVID-19 induced Pneumonia and Macrophage Activation Syndrome-Like Disease. Autoimmun Rev. 2020 Jun;19(6):102537. https://doi.org/10.1016/j.autrev.2020.102537.

Nicastri, M. et al. Efficacy of early physical therapy in severe Bell's palsy: a randomized controlled trial. Neurorehabil Neural Repair. Jul-Aug 2013;27(6):542-51. https://doi.org/10.1177/1545968313481280.

$\mathrm{Ou}, \mathrm{X}$. et al. Characterization of spike glycoprotein of SARS-CoV-2 on virus entry and its immune cross-reactivity with SARSCoV. Nat Commun 2020;11(1):1620. https://doi.org/10.1038/s41467-020-15562-9.

Pacheco, L. D. et al. Guillain-Barré Syndrome in Pregnancy. Obstet Gynecol. 2016 Nov;128(5):1105-1110. https://doi.org/ 10.1097/AOG.0000000000001716. 
Research, Society and Development, v. 10, n. 7, e18510716465, 2021

(CC BY 4.0) | ISSN 2525-3409 | DOI: http://dx.doi.org/10.33448/rsd-v10i7.16465

Peek, G. J. et al. Efficacy and economic assessment of conventional ventilatory support versus extracorporeal membrane oxygenation for severe adult respiratory failure (CESAR): a multicentre randomised controlled trial. Lancet. 2009 Oct 17;374(9698):1351-63. https://doi.org/10.1016/S01406736(09)61069-2.

Qeadan, F. et al. The risk of clinical complications and death among pregnant women with COVID-19 in the Cerner COVID-19 cohort: a retrospective analysis. BMC Pregnancy Childbirth. 2021 Apr 16;21(1):305. https://doi.org/10.1186/s12884-021-03772-y.

Ryan, G. A. et al. Clinical update on COVID-19 in pregnancy: A review article. J Obstet Gynaecol Res. 2020 Aug;46(8):1235-1245. https://doi.org/ 10.1111/jog.14321.

Sales, E. M. P. et al. Fisioterapia, funcionalidade e covid-19: revisão integrativa. Cadernos Esp. Ceará.2020, jan. jun.; 14(1) págs. 68-73ISSN: 18087329/1809-0893.

Shmorgun; D., Chan; W. S., \& Ray; J. G. Association between Bell's palsy in pregnancy and pre-eclampsia. QJM. 2002;95(6):359-62. https://doi.org/ 10.1093/qjmed/95.6.359.

Souza, A. S. Rolland; A., \& Melania; M. R. Maternal mortality by COVID-19 in Brazil. Rev. Bras. Saúde Matern. Infant., Recife, 21 (Supl. 1): S253-S256, fev., 2021. https://doi.org/10.1590/1806-9304202100S100014.

Tekin, A. B. et al. Guillain Barre Syndrome following delivery in a pregnant woman infected with SARS-CoV-2. J Clin Neurosci. 2021 Apr; 86:190-192. https://doi.org/10.1016/j.jocn.2021.01.028.

Van Den Berg, B. et al. Guillain-Barré syndrome: pathogenesis, diagnosis, treatment and prognosis. Nat Rev Neurol. 2014;10(8):469-82. https://doi.org/ $10.1038 /$ nrneurol.2014.121.

Varatharaj, A. et al. Neurological and neuropsychiatric complications of COVID-19 in 153 patients: a UK-wide surveillance study. Lancet Psychiatry. 2020 Oct;7(10):875-882. https://doi.org/10.1016/S2215-0366(20)30287-X.

Webb, S. et al. Guillain-Barré syndrome following COVID-19: a newly emerging post-infectious complication. BMJ Case Rep. 2020 Jun 14;13(6):e236182. https://doi.org/10.1136/bcr-2020-236182.

Zhang, L. et al. Severe acute respiratory syndrome coronavirus 2(SARS-CoV-2) infection during late pregnancy: a report of 18 patients from Wuhan, China. BMC Pregnancy Childbirth. 2020 Jul 8;20(1):394. https://doi.org/10.1186/s12884-020-03026-3.

Zhang, W. et al. The etiology of Bell's palsy: a review. J Neurol. 2020 Jul;267(7):1896-1905. https://doi.org/10.1007/s00415-019-09282-4. 